\title{
A graph-based machine learning approach to predicting digital lifecycle campaign engagement
}

\author{
Venkata Duvvuri \\ Department of Technology Leadership and Innovation, Purdue UniversityOracle Corporation
}

\begin{abstract}
Digital campaigns can be launched using a variety of channels, prominently display banner, social media, search, email and SMS. Quite a bit of research has been taken up to optimize such individual channel's performance. Due to integrated marketing needs to serve and nurture the customer over a lifetime, such campaigns are increasingly stringed together into a digital life cycle campaign (DLC) using graph structured representation, also called as marketing campaign orchestrations (MCO). Such DLC campaigns are quite complex and long in duration, including a variety of channels intermixed on multiple platforms. We suggest a novel way to measure engagement rates of such DLCs when prior historical campaign data is available for a well-defined marketing campaign orchestration. We additionally develop a way to predict performance of a new or modified DLC leveraging machine learning and graph techniques given historical DLC performances. We show that such predictions are more accurate in common scenarios when enterprises develop diverse DLCs.
\end{abstract}

\section{CCS CONCEPTS}

$\bullet$;

\section{KEYWORDS}

Computing methodologies

\section{ACM Reference Format:}

Venkata Duvvuri. 2021. A graph-based machine learning approach to predicting digital lifecycle campaign engagement. In 2021 The 5th International Conference on Machine Learning and Soft Computing (ICMLSC'21), fanuary 29-31, 2021, Virtual Event, Vietnam. ACM, New York, NY, USA, 6 pages. https://doi.org/10.1145/3453800.3453802

\section{INTRODUCTION}

Digital channels have increasingly become popular in nurturing and procuring customers for enterprises. While, traditional channels of print, banner and out-of-home still exist, the modern digital channels like Email, SMS, Push notification, Search, Display, Social media, Website etc. are becoming more efficient in nurturing and procuring customers due to data. Similar to Järvinen et. al. [1] we observe that with these digital channels, there is ease of availability of measurement data at various touch points that are normally not possible in traditional channels. Most research [2], [3], [4], [5], [6],

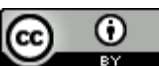

This work is licensed under a Creative Commons Attribution International 4.0 License.

ICMLSC'21, January 29-31, 2021, Virtual Event, Vietnam

(c) 2021 Copyright held by the owner/author(s).

ACM ISBN 978-1-4503-8761-3/21/01.

https://doi.org/10.1145/3453800.3453802
[7] has attempted to measure the efficacies of such individual digital channels, especially in search, banner, social media and email. We concur with Elina [16] that the broad levels of such efforts lead to the following categories of measurement metrics: a) engagement b) brand c) behavior d) financial.

Integrated marketing communication [8] is a phenomenon that's integral to several marketing enterprise tools [19]. As such, digital campaigns are increasingly stringed together as a series of intermixed campaigns to nudge the customer through the lifecycle from awareness to purchase. While, there have been measures of effectiveness of a single digital campaign, some methods are available for analysis of integrated campaigns specifically in Web Analytical tools [17], but not much research is available for the predicting the whole integrated (lifecycle) of digital campaign(s). This paper presents a novel way to predict performance of such digital lifecycle campaigns (DLC) based on historical data. Here we primarily focus on engagement metrics as these are the least common denominator for all digital individual channels. We defer measurement metrics for other categories like brand and financial to future research.

The paper is organized as follows: Section 2 discusses prior work, Section 3 high- lights measurements needed for a single digital lifecycle campaign, Section 4 provides an overview of a novel graph based machine learning Digital Lifecycle Campaign Engagement Model(GRAPHML_DLC_ENG_MODEL) to predict efficacy of a new digital lifecycle campaign, Section 5 presents experimentation and results for this approach when applied to real enterprise marketing datasets, Section 6 presents the pros and cons of this approach and finally Section 7 concludes the paper with future research directions. Table 1 reflects the nomenclature used in this paper.

\section{RELATED WORK}

Digital marketing amends itself to granular measurements [1] due to the scores of data that is collected at various touch points. Elina [16] observes that its challenging to get measurements (a.k.a. metrics) on efficacies of digital campaigns, due to multitudes of measuring strategies and contextual business relevance for some of them. Additionally, Steward [11] similarly observe that there are too many different kinds of such metrics and no consensus on when to apply particular metrics. Various researchers have their recommendation for measurements on various digital channels, like VanHoose [2] \& Danaher et. al. [7] for display banners, Ramos et. al. [3] for search engine marketing, Kelly [4], Kumar et. al. [5] \& Bhandari et. al. [6] for social media. Web channel is an interesting one, while not typically associated with digital marketing channels, but could pave a way on information for customers to learn further details of products and integrating measurement of various other digital channels into such Web analytics (WA) platforms. Järvinen et. al. [1] and Weishberg \& Kaushik [9] have extensively studied the use of web analytics as a tool to capture the performance of the 
Table 1: Nomenclature

\begin{tabular}{ll}
\hline Term & Description \\
\hline DLC & Digital Lifecycle Campaign \\
OR & Open Rate \\
CR & Click Rate \\
GRAPHML_DLC & Graph Machine Learning Digital Lifecycle Campaign Engagement \\
ENG_MODEL & Model \\
MCO & Marketing Campaign Orchestration \\
IMC & Integrated Marketing Communication \\
CX & Customer Experience \\
NDLC & New Digital Lifecycle Campaign (at design time) \\
NLP & Natural Language Processing \\
\hline
\end{tabular}

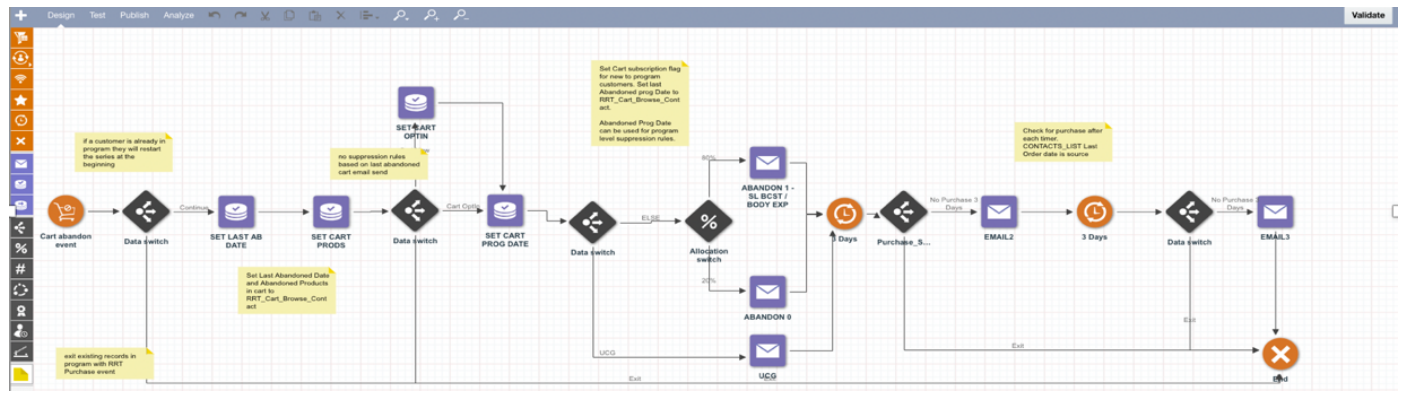

Figure 1: Digital Lifecycle Campaign

web channel and offer suggestions to improve business goals with WA. Still others, like Watróbski [10], have devised channel agnostic strategies for an individual digital channel's performance or looked at evaluating digital campaign measurements from a financial [11] and organization perspectives [12].

While, efficacies of individual channels have been researched, there is little to no research on measurement \& prediction of digital lifecycle campaign (DLC) that can have a mix variety of channels and can span over long durations and can be fairly complex. De Haan et. al. [18] lays out a framework for divvying up budget amongst multiple channels, typically referred to as channel attribution. But we address the converse problem, specifically aggregating and predicting the performance of the entire DLC rather than decomposing it. Fulgoni et. al. [13] has attempted to suggest needs and measurements across platforms (desktop, tablet, phone etc.) in a channel but does not offer lifecycle measurements guidance. Moreover, any approach that can address such complex campaigns with a mix of different platforms has to be generic and build on top of existing measurements strategies for digital channels. This paper presents such an audience weighted approach to measure performance of DLCs and builds on measurements for each individual digital channel. Additionally, using ML we predict the performance of a new or modified DLC, that is more effective in common scenarios like where customers have adopted IMC by building a diverse set of DLCs over a period of time.

\section{SINGLE DIGITAL LIFECYCLE CAMPAIGN}

Figure 1 shows a digital lifecycle campaign (DLC) as defined in an integrated marketing tool Oracle Responsys [19]. As per [19], "Oracle Responsys helps you manage, personalize, and orchestrate interactions across all channels to deliver timely, helpful messages in the moments that matter." Notably, the multiple campaigns from variety of channels can be stringed together in a workflow to orchestrate flow between several campaigns. This orchestration is so called as Marketing Campaign Orchestration (MCO).

\subsection{Methodology}

The performance measure of an existing single DLC forms the foundation of deriving the metric for a new or modified DLC. In this paper, we recommend engagement (opens, clicks etc.) as the measurement metric due to its universal availability across all channels. While, there could be many methods to combine the metrics of individual campaigns in a DLC to arrive at an overall engagement metric, we formulate a simple method based on weighted averages of audience's input to each of the campaigns in a DLC, as we believe that overall ratio of audience received by a campaign serves as a leading indicator of its influence in the entire DLC. We further integrate multiple channels by divvying up channel metrics based on intuitive assumptions (functions) of their weightage in a multiple channel DLC. For example, a uniform weighting function gives equal weights for all channels.

Mchannel metrics per channel: We define a weighted average method to arrive at the metrics ( $\mathrm{M}$ - opens, clicks etc.) per channel as 


$$
\begin{aligned}
& \mathrm{M}_{\text {channel }}=\frac{\sum_{1}^{u} W_{s u} * S u_{-} \text {channel }}{\sum_{1}^{u} W_{s}}=\frac{W_{S 1} * S 1_{s m s}+\cdots+W s u * S u_{-} s m s}{1} \\
& \mathrm{~W}_{\mathrm{su}}=\frac{\sum \text { send enactments in stage for all channels }}{\sum \text { send enactments in any stage for all channles }} \\
& \mathrm{S}_{\mathrm{u}_{-} \text {channel }}=\frac{\sum \text { distict metric }(\text { opens,clicks } \text { etc) } \text { enactments in stage per channel }}{\sum \text { send enactment in stage per channel }} \\
& \mathrm{M}_{\text {overall }}=\frac{\sum_{1}^{c} A_{m \_} \text {channel } * \text { Mchannel }}{\sum_{1}^{c} A_{m} \text { channel }}=\frac{A_{m \_s m s} * M_{\text {sms }}+A_{m \_p u s h} * M_{\text {push }}+A_{m \_ \text {email }} * M_{\text {email }}}{1} \\
& \mathrm{~A}_{\mathrm{m}_{-} \text {channel }}=\frac{\text { Smetric (opens,clicks,cvr) enactments in program (all stages) per channel }}{\sum \text { metric (opens,clicks,cvr) enactments in program (all stages) in all channel }}
\end{aligned}
$$

Figure 2: Single Digital Lifecycle Campaign Measurement

in Figure 2. Here the weights are decided by a proportion of sends (a.k.a audiences) in that stage (a.k.a campaign) compared to all stages combined in $\mathrm{P}$ (graph). Let the DLC be described by a graph $\mathrm{P}$, also known as MCO (Marketing Campaign Orchestration). Let each of the nodes in P be referred to as "stages or campaigns". Let an "enactment" represent a string of consecutive flow of a user's activity in $\mathrm{P}$, from start to finish over a period of time. Weight Wsu_channel is the proportion of send enactments (audiences) in stage $u$ per channel as compared to the combined send enactments in entire P. Let Su_channel be per channel engagement metric (Open rate, Click rate etc.) for each of u stages in MCO (P) in the last D days. Moverall DLC metric for all channels: Let there be $c$ channels like SMS (channel_1), push, display banner, ......, email (channel_c).We combine channel metrics (Mchannel) by a weight Am_channel calculated as the proportion of the channel metric to the total metric for all channels in P.

\section{NEW DIGITAL LIFECYCLE CAMPAIGN}

We derive a machine learning (ML) approach to predict the engagement metric for a new or modified digital lifecycle campaign (NDLC) at design time. The main features used for this purpose is the campaign $\mathrm{MCO}$ graph structure (P, represented as a vector as in Section 4.1) and campaign meta data like campaigns names, node labels etc. (represented as a vector in Section 4.2). The crux of our approach is to use graph similarities to derive which existing graphs are similar to new ones. While, this may be enough to get reasonable matches, sometimes we need to look at the details of the campaigns within a graph. We augment first step (of using graph similarities) with campaign similarities as a pruning step to weed out matches that are not relevant. Thus, we arrive at a good set of matches for a NDLC from historical DLCs. Our engagement for the NDLC can be calculated from historical DLC's using weighted averages or a similar aggregating function.

\subsection{Graph similarities}

Typical MCO's have graph structures as in Figure 1, with nodes (campaigns, logic elements etc.) and edges (connectors etc.) that lend themselves to using machine learning techniques from a meaningful summary representation (a.k.a embeddings). graph2vec [14] is a recent neural network-based technique that generates machine learning representations of such graphs using unsupervised learning. It can accommodate graphs of varying sizes and leverage non-linear neural network algorithm at its core. Narayanan et. al. [14] demonstrates that this is superior to traditional methods of representing graphs. After generating such graph2vec representations, we perform cosine similarities on each graph's vectors to infer similar graphs.

\subsection{Campaign similarities}

Typically, a graph $\mathrm{P}$ has several individual campaign nodes that drive messaging to the end customers. These nodes have several descriptive text information or other meta data characteristics that can be leveraged to calculate how campaigns are similar within two Ps. In general, such campaign information can some take various forms, but we focus on textual data for campaign due to its general availability when marketers design DLCs. Example meta data is a textual label for the campaign node in $\mathrm{P}$, campaign names given to them by marketers or long description added to each campaign node. We leverage such data by performing natural language processing (NLP) by representing each $\mathrm{P}$ as a concatenated single sentence of such text information. We then represent these sentences using aggregated word2vec [15] representations in a model trained over all the words in all Ps that an enterprise has built. Since, word2vec is a real typed vector representation, we can perform cosine similarity over any two or more sentences and find out which P's are close enough to a new or modified graph $\mathrm{P}^{*}$ within a threshold.

\subsection{Graph ML digital lifecycle campaign engagement model (GRAPHML_DLC_ENG_MODEL)}

In summary, as in Figure 3, our approach for predicting engagement metrics of a new or modified DLC (NDLC) at design time is based on finding similar DLCs. The graph for each DLC is captured 
by a 108 vector embedding using graph2vec, connoted as an embedding in Figure 3. The embeddings are pre-calculated for every historical DLC during training time after a series of preprocessing steps a) extraction of DLC graph P b) extraction of campaign text $\&$ other text data c) identification of nodes and edges. These are then fed to graph2vec neural network model as highlighted in Section 4.1 During inference time, when a NDLC is being designed or modified, for its predictions, we first calculate its embedding using graph2vec. Then using cosine similarities over all historical DLC embeddings in enterprise, we arrive at an initial list of candidate matches DLCs $(C)$ that are structurally similar. In next step, we account for campaign similarities using textual information (Text labels, descriptions, campaign labels) obtained from one of the pre-processing steps above. Then using word2vec model over such text we can build campaign embeddings as highlighted in Section 4.2. Then we use NLP over such text to capture how an NDLC (N) is similar to each of the candidate DLCs (C) and prune those that are not relevant within a threshold. Thus, the final set $(\mathrm{F})$ incorporates both graph similarities and campaign similarities. The engagement rate (click rates or open rates) metrics for the NDLC is derived using a weighted aggregate or a statistically function approximator over such candidate matched DLCs in set F. Each candidate DLC engagement metric in $\mathrm{F}$ is calculated as highlighted in Section 3. Figure 3 represents a workflow of this graph ML algorithm/model (GRAPHML_DLC_ENG_MODEL), including training and prediction flows.

\section{EXPERIMENTATION}

\subsection{Dataset}

For our evaluation we have a test setup of three real big accounts in Oracle CX Marketing product from three big retailers with total of $100+$ big (having 4+ nodes in each graph) DLCs in each dataset. Each dataset is from a top enterprise customer for Oracle CX marketing products [19] with 100's of DLCs built over several years of product adoption. These are historical DLCs we know the performance of each one of them over a period, including the NDLCs (each DLC serves as an NDLC in turn for experimentation evaluation).

\subsection{Distribution}

As per above audience weighted method to calculate DLC engagement metric, Figure 4 shows the distribution (number of graphs or Ps binned over open rates or click rates) for multiple DLC engagement metrics for three different real enterprise digital marketing datasets (dataset1, dataset2, dataset3). From Figure 4 we observe that dataset2 has a wide distribution of DLC opens rates and seems more unimodal. Clicks are unimodal too, but more clustered around mode. In dataset1, the distribution of opens is non-modal, and is quite flat. The distributions of click rates is quite wide along with a single mode. Finally, the distribution of opens and clicks for dataset3 is quite narrow and strongly unimodal. Thus, as noted from above variety of DLC metrics and the marketers need to build variety of DLCs in same or various enterprises, the task of a predicting a new of modified DLC engagement metric using a common model is quite interesting.

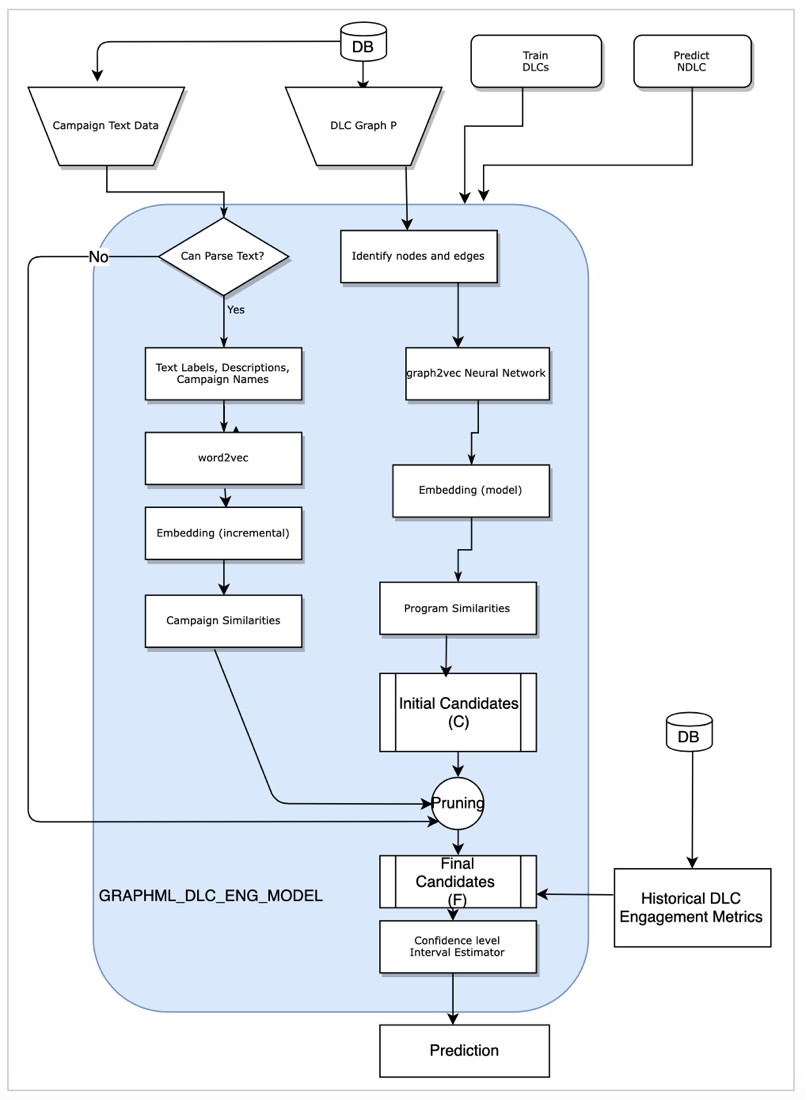

Figure 3: GRAPHML_DLC_ENG_MODEL for predicting NDLC performance

\subsection{Results}

For each DLC we apply above GRAPHML_DLC_ENG_MODEL model and calculate predictions of NDLC engagement metrics (OR, $\mathrm{CR})$. We generate interval estimates of click and open rate engagement metrics from final matched list (F) using statistical inference approximation with a confidence level of 75\% CL (a deviation from typical $90-95 \%$ due to sparsity of historical measurements in typical DLC's in [19]). This interval range results are reported as the final prediction for a NDLC. We consider a prediction accurate or correct if the actual or historical engagement metric is within the prediction range. In GRAPHML_DLC_ENG_MODEL there are several hyperparameters like cosine similarity thresholds (cosine_nlp), graph embedding similarity thresholds (cosine_graph_threshold) etc. as shown in Table 3

We compare our model with two intuitive models a) Best or Educated guess b) Statistical (benchmark) model. In best guess a marketer took an educated guess about the performance of a new DLC based on the distribution of the metrics as shown in Figure 4. This performance is shows as "best guess range". As observed in Table 2, except for trivial cases like in dataset3, where the engagements distribution is fairly narrow, this model won't work for most enterprises. Thus, the problem of predictions is non-trivial. 

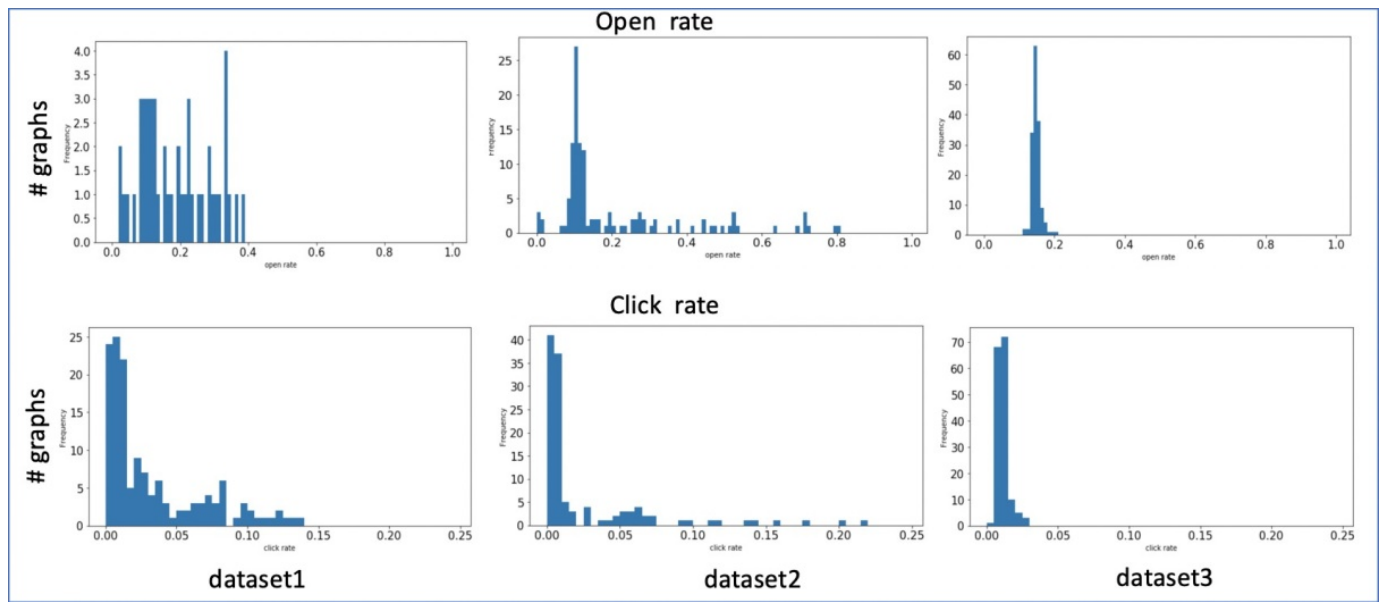

Figure 4: Distributions of three enterprise datasets DLC engagement metrics

Table 2: GRAPHML_DLC_ENG_MODEL Model comparison with statistical models

\begin{tabular}{|c|c|c|c|c|}
\hline Model dataset 1 & & dataset2 & dataset3 & aggregate 3 \\
\hline & $\begin{array}{r}\text { OR/ CR } \\
\text { accuracy }\end{array}$ & $\begin{array}{l}\mathrm{OR} / \mathrm{CR} \\
\text { accuracy }\end{array}$ & $\begin{array}{l}\text { OR/ CR } \\
\text { accuracy }\end{array}$ & $\begin{array}{l}\text { datasets } \mathrm{OR} / \mathrm{CR} \\
\text { accuracy }\end{array}$ \\
\hline Educated Guess & $23 \% / 16 \%$ & $8 \% / 14 \%$ & $63 \% / 5 \%$ & $31 \% / 11 \%$ \\
\hline Statistical (Benchmark) & $23 \% / 17 \%$ & $34 \% / 90 \%$ & $42 \% / 49 \%$ & $33 \% / 52 \%$ \\
\hline $\begin{array}{l}\text { GRAPHML_DLC_ENG_ } \\
\text { MODEL }\end{array}$ & $46 \% / 57 \%$ & $62 \% / 65 \%$ & $46 \% / 44 \%$ & $51 \% / 55 \%$ \\
\hline Lift $^{*}$ & $100 \% / 235 \%$ & $82 \% /-28 \%$ & $10 \% /-10 \%$ & $55 \% / 6.4 \%$ \\
\hline $\begin{array}{l}\text { Model OR/CR prediction } \\
\text { range difference from } \\
\text { benchmark's range }\end{array}$ & $0 \% / 0 \%$ & $0 \% / 0 \%$ & $+0.6 \% /+0.1 \%$ & - \\
\hline
\end{tabular}

Table 3: GRAPHML_DLC_ENG_MODEL Hyperparameters

\begin{tabular}{ll}
\hline GRAPHML_DLC_ENG_MODEL & Parameter value \\
\hline Confidence & 0.75 \\
cosine_nlp_threshold & 0.37 \\
cosine_graph_threshold & 0.50 \\
outlier_threshold & 1.5 \\
benchmark_range_in_std_dev & 1 \\
no_similar_graphs & 5 \\
\hline
\end{tabular}

* improvement in GRAPHML_DLC_ENG_MODEL OR/CR over statistical benchmark

The second model we compare to is a statistical benchmark that estimates the NDLC performance as mean 1 standard deviation of a normally approximated curve for all historical engagement distributions for all DLCs in enterprise. In general, for normal distribution, ones can expect a $38 \%$ accuracy using probability density over 1 standard deviation. But, since the distributions are not normal most times, we see from Table 2 that the accuracy ranges from $17 \%$ to $49 \%$, except for an outlier in dataset 2 . Thus, this model serves as the statistical benchmark for evaluating our model to beat.
As per the results shown in Table 2, in aggregate GRAPHML_DLC_ENG_MODEL model performs 55\%/6.4\% better than statistical benchmark model when prediction ranges are similarly around 1 standard deviation as compared to the statistical benchmark model range. For open rates, our model performs $82 \%$ to $100 \%$ better, when enterprises have diverse and non-uniform distributed DLC's, as in dataset1 and dataset2. For click rates, when DLCs are also diversely distributed as in dataset1, we show a significant lift (235\%). The model performs at par with statistical benchmark when the distributions are normal or near normal, like in dataset3. For dataset2, click rates have kurtosis 
Table 4: No Match accuracy

\begin{tabular}{lll}
\hline Model for no Match & Open Rate Accuracy & Click Rate Accuracy \\
\hline Model2 Accuracy & $21 \% / 66 \% / 47 \%$ & $78 \% / 0 \% / 51 \%$ \\
\hline
\end{tabular}

Table 5: Tiered GRAPHML_DLC_ENG_MODEL model performance and match rates

\begin{tabular}{llll}
\hline Distribution & $\begin{array}{l}\text { Match rate } \\
\text { dataset1/dataset2/ dataset3 }\end{array}$ & $\begin{array}{l}\text { Open Rate Accuracy } \\
\text { dataset1/dataset2/ dataset3 }\end{array}$ & $\begin{array}{l}\text { Click Rate Accuracy } \\
\text { dataset1/dataset2/ dataset3 }\end{array}$ \\
\hline $\begin{array}{l}\text { Model1 } \\
\text { Match }\end{array}$ & $77 \% / 80 \% / 85 \%$ & $50 \% / 62 \% / 55 \%$ & $57 \% / 62 \% / 58 \%$ \\
$\begin{array}{l}\text { Model1 } \\
\text { No match }\end{array}$ & $23 \% / 20 \% / 15 \%$ & & \\
\hline
\end{tabular}

greater than 3, and are leptokurtic, having a slender distribution. Hence, in this special scenario, the statistical model outperforms our model. We expect such cases to rarely be the norm, hoping that enterprises build different and diverse DLCs over a longer period of marketing activities.

\section{PROS AND CONS}

GRAPHML_DLC_ENG_MODEL assumes a historical diverse set of DLCs built by enterprises, with engagement metrics gathered regularly that can be explored by our models for predictions. Without a base set of such DLC's, our approach may not function well. In our model, two machine learning approaches (graph similarities and campaigns similarities) are chained together. This sometimes leads to no or fewer reasonable matches, when applying both the criteria. Hence, instead of predicting no metrics when the marketer could be in limbo, we can build two models, a tighter one as in model1 (graph + campaign similarities), and a less constricting one as in model 2 (graph similarities only). This ensures that there are reasonable results for predictions in all or most cases. As shown in Table $4 \& 5$, on an average, model 1 performs at $57 \%$ accuracy, and model 2 performs at $45 \%$ accuracy. Though less accurate for model 2 the results are still valuable to enterprises. In our experimentation, in aggregate, we observe that model 1 successfully finds matches $81 \%$ of the times and remaining $19 \%$ of the times model 2 matches have to be used.

\section{CONCLUSION}

Digital campaigns have existed for the past two decades and in various forms: display, email, social, web, SMS and push etc. Increasingly these campaigns are stringed into digital lifecycle campaigns to achieve integrated marketing. While, techniques exist to measure the performance of individual campaigns, measurement of DLC has not been well addressed and is fairly new in industry. Due to machine learning techniques it has become easy to predict the performance of a new DLC at design time, leveraging the calculation of engagement metrics of historical DLCs. We propose a novel intuitive way to calculate the performance of an existing DLC using weighted averages. We also lay out a novel graph-based machine learning approach to predict performance of new DLC.
We demonstrate through experimentation over real enterprise marketing datasets that such a model beats a statistical benchmark by $55 \%$ in common scenarios in enterprises.

\section{REFERENCES}

[1] Järvinen J, Karjaluoto H. 2015. The use of Web analytics for digital marketing performance measurement. Industrial Marketing Management. 50:117-127. Available from: 10. 1016/j.indmarman.2015.04.009; https://dx.doi.org/10.1016/j. indmarman.2015.04.009.

[2] VanHoose, D. 2011. D. E-Commerce Economics (2nd ed.). Abingdon: Routledge.

[3] Ramos A, Cota S. 2014. Search engine marketing. New York: McGraw-Hill.

[4] Kelly N. 2013. How to measure social media a step-by-step guide to developing and accessing social media roi. Indianapolis: QUE.

[5] Kumar A, Bezawada R, Rishika R, et al. From Social to Sale: 2016. The Effects of Firm- Generated Content in Social Media on Customer Behavior. Journal of Marketing; 80(1):7-25. Available from: 10.1509/jm.14.0249; https://dx.doi.org/10. 1509/jm.14.0249.

[6] Bhandari, R., Gordon, J. and Umblijs, A., 2012. Getting beyond the buzz: Is your social media working. Financial Times.

[7] Danaher PJ, Lee J, Kerbache L. Optimal Internet Media Selection. Marketing Science. 2010;29(2):336-347. Available from: 10.1287/mksc.1090.0507;https://dx. doi.org/10.1287/ mksc.1090.0507.

[8] Kitchen PJ, Burgmann I. 2010. Integrated marketing communication. Wiley international end-cyclopedia of marketing.

[9] Waisberg D, Kaushik A. 2009. Web Analytics 2.0: empowering customer centricity. The original Search Engine Marketing Journal;2(1):5-11.

[10] Watróbski, Jankowski, and Ziemba. "MULTISTAGE PERFORMANCE MODELLING IN DIGITAL MARKETING MANAGEMENT." Economics \& Sociology 9.2. 016.: 101-25. Web.

[11] Stewart DW. 2009. Marketing accountability: Linking marketing actions to financial results. Journal of Business;62(6):636-679.

[12] Fatin T, Rahman N. 2020. Measuring Digital Marketing Performance. International Journal of Applied Management Theory and Research.2(1):1-15. Available from: 10.4018/ ijamtr.2020010101; https://dx.doi.org/10.4018/ijamtr.2020010101.

[13] Fulgoni G, Lipsman A. Numbers, 2014. Please: Digital Game Changers: How Social Media Will Help Usher in the Era of. Journal of Advertising Research. 54(1).

[14] Narayanan, Annamalai, et al. 2017. "graph2vec: Learning distributed representations of graphs." arXiv preprint arXiv:1707.05005.

[15] CHURCH KW. 2017. Word2Vec. Natural Language Engineering. 23(1):155162. Available from: 10.1017/s1351324916000334;https://dx.doi.org/10.1017/ s1351324916000334.

[16] Gaitniece, Elina. 2018. "Digital Marketing Performance Evaluation Methods." CBU International Conference Proceedings. Vol. 6.

[17] M. Mitova. 2017. "UTM Builders Guide". Retrieved from https://www. campaigntrackly.com/google-utm-builder-guide/

[18] De Haan, Evert, Thorsten Wiesel, and Koen Pauwels. 2016. "The effectiveness of different forms of online advertising for purchase conversion in a multiplechannel attribution framework." International Journal of Research in Marketing 33.3. 491-507.

[19] "Oracle Responsys". 2020. Retrieved from https://www.oracle.com/cx/marketing/ campaign-managem 